\title{
Price of Asymptotic Safety
}

\author{
Andrew D. Bond ${ }^{*}$ and Daniel F. Litim ${ }^{\dagger}$ \\ Department of Physics and Astronomy, University of Sussex, Brighton, BN1 9QH, United Kingdom
}

(Received 1 February 2018; revised manuscript received 17 April 2019; published 29 May 2019)

\begin{abstract}
All known examples of four-dimensional quantum field theories with asymptotic freedom or asymptotic safety at weak coupling involve non-Abelian gauge interactions. We demonstrate that this is not a coincidence: No weakly coupled fixed points, ultraviolet or otherwise, can be reliably generated in theories lacking gauge interactions. Implications for particle physics, critical phenomena, and conformal field theory are indicated.
\end{abstract}

DOI: 10.1103/PhysRevLett.122.211601

Introduction.-A turning point in the understanding of high-energy physics has been the discovery of asymptotic freedom in non-Abelian gauge theories [1,2]. It ensures that certain renormalizable quantum field theories remain predictive in the high-energy limit where couplings become free [3-5]. Non-Abelian gauge fields are decisive for this to happen: Without them, asymptotic freedom cannot be achieved in any theory involving Dirac fermions, photons, or scalars [6].

In the absence of asymptotic freedom, particle theories are generically plagued by divergences and a breakdown of predictivity in the high-energy limit. Some such theories, however, remain well defined thanks to strict cancellations at the quantum level $[7,8]$ and display "asymptotic near freedom" [9] or "asymptotic safety" [10] at high energies. Thereby, running couplings achieve an interacting fixed point under the renormalization group evolution, which serves as an anchor for short-distance quantum fluctuations [11]. General theorems for asymptotic safety are available for weakly coupled gauge-matter theories [8] and cover simple [7,12], semisimple [13], and supersymmetric [14] gauge theories and extensions beyond the standard model [15]. For studies also involving quantum gravity, see Refs. [16-32], and references therein.

It appears that all known examples of four-dimensional particle theories with asymptotic freedom or asymptotic safety at weak coupling involve non-Abelian gauge interactions. It is the purpose of this Letter to demonstrate that this is not a coincidence: No weakly interacting fixed points, ultraviolet or otherwise, can be reliably generated in theories lacking gauge interactions. Partial results in support of our claim have been made available in Refs. [6,8].

Published by the American Physical Society under the terms of the Creative Commons Attribution 4.0 International license. Further distribution of this work must maintain attribution to the author(s) and the published article's title, journal citation, and DOI. Funded by SCOAP.
Here, we provide the missing pieces, which are, on the one hand, an extension of the Coleman-Gross theorem [6] and a no-go-theorem for weakly interacting fixed points in nongauge theories, on the other. Taken together, non-Abelian gauge interactions are the unique price for particle theories to remain strictly perturbative and predictive at asymptotically high energies and to display weakly coupled fixed points at low energies.

Price of asymptotic freedom.-To establish our claim, we first revisit asymptotic freedom of general, renormalizable particle theories in four dimensions involving gauge fields, fermions, or scalars. Without loss of generality, we limit the analysis to the canonically marginal interactions which are the gauge, the Yukawa, and the scalar selfcouplings $\left\{g_{i}, \mathbf{Y}_{I J}^{A}, \lambda_{A B C D}\right\}$, respectively. We assume canonically normalized kinetic terms with gauge couplings $g_{i}$ for each gauge factor. Our conventions for the most general Yukawa and scalar couplings are

$$
\begin{aligned}
L_{\mathrm{Yuk}} & =-\frac{1}{2}\left(\mathbf{Y}_{J K}^{A} \Phi^{A} \Psi_{J} \Psi_{K}+\text { H.c. }\right), \\
L_{\mathrm{pot}} & =-\frac{1}{4 !} \lambda_{A B C D} \Phi^{A} \Phi^{B} \Phi^{C} \Phi^{D},
\end{aligned}
$$

where $\Psi_{J}$ denote Weyl fermions and $\Phi^{A}$ real scalars. Matter fields may be charged under the gauge groups.

Next, we turn to quantum effects and the renormalization group running of couplings. The point in coupling space where all couplings vanish, the free theory, is always a fixed point of the renormalization group. Then, for any theory to be free at asymptotically high energies, the free fixed point must be ultraviolet and the beta functions negative for sufficiently small couplings:

$$
\mu \partial_{\mu}(g, Y, \lambda)<0
$$

with $\mu$ the renormalization group scale. After scaling the loop factor into the couplings, as we shall consistently do throughout, the one-loop gauge beta function is [1-3] 


$$
\mu \partial_{\mu} g_{i}=B_{i} g_{i}^{3}, \quad B_{i}=-\frac{11}{3} C_{2}^{G_{i}}+\frac{2}{3} S_{2}^{F_{i}}+\frac{1}{6} S_{2}^{S_{i}} .
$$

Non-Abelian gauge fields contribute negatively to the oneloop coefficient $\left(B_{i}\right)$, proportionally to the Casimir of the gauge group $\left(C_{2}^{G_{i}}\right)$. Matter fields contribute positively and proportionally to their Dynkin indices $\left(S_{2}\right)$. The main feature of non-Abelian theories is that (3) can have either sign. A negative one-loop coefficient is known to offer the unique key for asymptotic freedom [3-5]. Below, we demonstrate that non-Abelian fluctuations, in particular, the smallness of the one-loop coefficient for suitable matter and irrespective of the sign of (3), also provide the unique key for weakly interacting fixed points including asymptotic safety of theories with $B_{i}<0$.

Coleman-Gross theorem revisited.-To clarify the role of gauge field fluctuations for asymptotic freedom, we first revisit the Coleman-Gross theorem [6]. It states that a nongauge theory of scalars with or without Dirac fermions cannot become asymptotically free. To cover the most general setting, we extend the theorem towards Weyl fermions. It is convenient to view the Yukawa couplings as symmetric matrices $\mathbf{Y}^{A}$ in the fermion indices $\left(\mathbf{Y}^{A}\right)_{J K} \equiv \mathbf{Y}_{J K}^{A}$. Their running with momentum scale $\mu$ at the leading order in the perturbation theory reads $[33,34]$

$\mu \partial_{\mu} \mathbf{Y}^{A}=\frac{1}{2}\left(\overline{\mathbf{Y}_{2}^{F}} \mathbf{Y}^{A}+\mathbf{Y}^{A} \mathbf{Y}_{2}^{\boldsymbol{F}}\right)+Y_{2}^{S A B} \mathbf{Y}^{B}+2 \mathbf{Y}^{B} \mathbf{Y}^{A \dagger} \mathbf{Y}^{B}$,

where the bar denotes complex conjugation and summation over repeated indices is implied. We have also introduced the quadratic combinations $\mathbf{Y}_{2}{ }_{J K}^{A B}=\frac{1}{2}\left(\mathbf{Y}^{A \dagger} \mathbf{Y}^{B}+\mathbf{Y}^{B \dagger} \mathbf{Y}^{A}\right)_{J K}$ alongside $\mathbf{Y}_{2}^{F}{ }_{J K} \equiv \mathbf{Y}_{2 J K}^{A A}$ and $Y_{2}^{S A B} \equiv \mathbf{Y}_{2}{ }_{J J}^{A B}$. The first and second terms in (4) arise from the wave function renormalization of the fermion and scalar propagators, whereas the last term stems from vertex corrections. We note that the Yukawa couplings and their flows (4) transform as tensors under a change of base, i.e., general linear transformations of the fields which leave (1) invariant.

We shall now focus our attention on the flow for the sum of the squared absolute values of all Yukawa couplings:

$$
\begin{aligned}
\mu \partial_{\mu} \operatorname{Tr} & \left(\mathbf{Y}^{A \dagger} \mathbf{Y}^{A}\right) \\
= & \operatorname{Tr}\left[\left(\overline{\mathbf{Y}_{2}^{F}}\right)^{2}\right]+\operatorname{Tr}\left[\left(\mathbf{Y}_{\mathbf{2}}^{\boldsymbol{F}}\right)^{2}\right]+4 \operatorname{Tr}\left(\mathbf{Y}^{A \dagger} \mathbf{Y}^{B} \mathbf{Y}^{A \dagger} \mathbf{Y}^{B}\right) \\
& +\operatorname{Tr}\left(\mathbf{Y}^{A \dagger} \mathbf{Y}^{B}\right)\left[\operatorname{Tr}\left(\mathbf{Y}^{A \dagger} \mathbf{Y}^{B}\right)+(A \leftrightarrow B)\right],
\end{aligned}
$$

for if we are to have all Yukawa beta functions negative, then this combination must be negative as well. We emphasize that the flow (5) and the conclusions drawn from it are independent of the choice of field base. A lower bound for (5) follows by using that $\operatorname{Re} z^{2} \leq z^{*} z$ for any complex number $z$, whence

$$
\operatorname{Tr}\left(\mathbf{Y}^{A \dagger} \mathbf{Y}^{B}\right) \operatorname{Tr}\left(\mathbf{Y}^{A \dagger} \mathbf{Y}^{B}\right) \leq \operatorname{Tr}\left(\mathbf{Y}^{A \dagger} \mathbf{Y}^{B}\right) \operatorname{Tr}\left(\mathbf{Y}^{B \dagger} \mathbf{Y}^{A}\right) .
$$

Next, we introduce the three real trace invariants $T_{1}=\operatorname{Tr}\left[\left(\mathbf{Y}_{\mathbf{2}}^{\boldsymbol{F}}\right)^{2}\right]=\operatorname{Tr}\left[\left(\overline{\mathbf{Y}_{2}^{\boldsymbol{F}}}\right)^{2}\right], T_{2}=\operatorname{Tr}\left(\mathbf{Y}^{A \dagger} \mathbf{Y}^{B} \mathbf{Y}^{A \dagger} \mathbf{Y}^{B}\right)$, and $T_{3}=\operatorname{Tr}\left(\mathbf{Y}^{A \dagger} \mathbf{Y}^{B}\right) \operatorname{Tr}\left(\mathbf{Y}^{A \dagger} \mathbf{Y}^{B}\right)$. By definition, $T_{2}$ may have either sign while $T_{1}, T_{3} \geq 0$. In terms of these, and together with (6), we find that the Yukawa beta function (5) is bounded from below:

$$
\mu \partial_{\mu} \operatorname{Tr}\left(\mathbf{Y}^{A \dagger} \mathbf{Y}^{A}\right) \geq 2\left(T_{1}+T_{2}\right)+2\left(T_{2}+T_{3}\right) .
$$

Recalling that the Yukawa couplings are symmetric in the fermionic indices, we rearrange the sums as follows:

$$
\begin{aligned}
T_{1}+T_{2} & =\mathbf{Y}_{J K}^{A \dagger} \mathbf{Y}_{K L}^{A} \mathbf{Y}_{L M}^{B \dagger} \mathbf{Y}_{M J}^{B}+\mathbf{Y}_{J K}^{A \dagger} \mathbf{Y}_{K L}^{B} \mathbf{Y}_{L M}^{A \dagger} \mathbf{Y}_{M J}^{B} \\
& =\mathbf{Y}_{J K}^{A \dagger} \mathbf{Y}_{M J}^{B}\left(\mathbf{Y}_{K L}^{A} \mathbf{Y}_{L M}^{B^{\dagger}}+\mathbf{Y}_{K L}^{B} \mathbf{Y}_{L M}^{A \dagger}\right) \\
& =\frac{1}{2}\left(\mathbf{Y}_{J K}^{A \dagger} \mathbf{Y}_{M J}^{B}+\mathbf{Y}_{J K}^{B^{\dagger}} \mathbf{Y}_{M J}^{A}\right)\left(\mathbf{Y}_{K L}^{A} \mathbf{Y}_{L M}^{B^{\dagger}}+\mathbf{Y}_{K L}^{B} \mathbf{Y}_{L M}^{A \dagger}\right) \\
& =2 \mathbf{Y}_{2 K M}^{A B} \mathbf{Y}_{2 M K}^{A B}=2 \overline{\mathbf{Y}_{2 M K}^{A B}} \mathbf{Y}_{2 M K}^{A B}, \\
T_{2}+T_{3} & =\mathbf{Y}_{J K}^{A} \mathbf{Y}_{K L}^{B \dagger} \mathbf{Y}_{L M}^{A} \mathbf{Y}_{M J}^{B^{\dagger}}+\mathbf{Y}_{J K}^{A} \mathbf{Y}_{K J}^{B^{\dagger}} \mathbf{Y}_{L M}^{A} \mathbf{Y}_{M L}^{B^{\dagger}} \\
& =\mathbf{Y}_{J K}^{A} \mathbf{Y}_{L M}^{A}\left(\mathbf{Y}_{K J}^{B \dagger} \mathbf{Y}_{M L}^{B^{\dagger}}+\mathbf{Y}_{K L}^{B^{\dagger}} \mathbf{Y}_{M J}^{B^{\dagger}}\right) \\
& =\frac{1}{2}\left(\mathbf{Y}_{K J}^{A} \mathbf{Y}_{M L}^{A}+\mathbf{Y}_{K L}^{A} \mathbf{Y}_{M J}^{A}\right)\left(\mathbf{Y}_{K J}^{B \dagger} \mathbf{Y}_{M L}^{B^{\dagger}}+\mathbf{Y}_{K L}^{B \dagger} \mathbf{Y}_{M J}^{B^{\dagger}}\right)
\end{aligned}
$$

As is evidenced by the explicit expressions, both (8) and (9) are sums of absolute values squared and therefore manifestly semipositive definite:

$$
\begin{aligned}
& T_{1}+T_{2} \geq 0, \\
& T_{2}+T_{3} \geq 0 .
\end{aligned}
$$

Most importantly, the bounds (10) dictate positivity for the flow (7) close to the Gaussian,

$$
\mu \partial_{\mu} \operatorname{Tr}\left(\mathbf{Y}^{A \dagger} \mathbf{Y}^{A}\right) \geq 0
$$

and establish that asymptotic freedom is unavailable. Had we substituted Weyl by Dirac fermions in (1), we would have found the lower bound $\mu \partial_{\mu} \operatorname{Tr}\left(\mathbf{Y}^{A \dagger} \mathbf{Y}^{A}\right) \geq 2 T_{1}+$ $4\left(T_{2}+T_{3}\right)$, instead of (7). For theories with Dirac fermions only, the non-negativity of $T_{1}$ together with $T_{2}+$ $T_{3}>0$ is sufficient to conclude the absence of asymptotic freedom [6]. Clearly, the bounds for Weyl and Dirac fermions are inequivalent: While the former entail the latter, the converse is not true.

One might wonder whether scalar self-interactions may upset the conclusion. Scalar couplings contribute to the Yukawa beta function starting at two-loop order. Therefore, if they were to reliably generate asymptotic freedom, they must do so along a renormalization group trajectory where they are parametrically larger than the Yukawa couplings. Assuming this to be the case, we can then ignore the 
Yukawa contribution to the running of the quartics. In other words, the scalar sector must become asymptotically free in its own right. This, however, is known to be impossible [6]. We reproduce here the line of reasoning, as some of this is needed later.

To leading order in perturbation theory, a scalar theory with quartic interactions (1) has the beta function [4]

$$
\beta_{A B C D}=\frac{1}{8} \sum_{\{A B C D\}} \lambda_{A B E F} \lambda_{E F C D}
$$

where $\beta_{A B C D} \equiv \mu \partial_{\mu} \lambda_{A B C D}$ with $\lambda$ fully symmetric in its indices and the sum running over all permutations. For clarity, in the following we shall write out any index sums explicitly. Vacuum stability requires that for each $A$ we must have $\lambda_{A A A A} \geq 0$, or else the potential becomes unbounded in the $\phi_{A}$ direction. Together with (12), we have

$$
\beta_{A A A A} \propto \sum_{B, C} \lambda_{A A B C} \lambda_{A A B C} \geq 0
$$

showing that vacuum stability is incompatible with asymptotic freedom, for which we would need this beta function to be negative, (2). Let us then switch off all such couplings identically, $\lambda_{A A A A}(\mu)=0$. In this scenario, their flows and all couplings appearing on the right-hand side of (13) have to vanish, or else a nonzero value for $\lambda_{A A A A}$ is generated by fluctuations. Specifically, taking $B=C$, it follows that $\lambda_{A A B B}(\mu)=0$ at all scales, which again necessitates $\beta_{A A B B}=$ 0 . Since these beta functions are the sums of squares,

$$
\beta_{A A B B}=\beta_{A B A B} \propto \sum_{C, D} \lambda_{A B C D} \lambda_{A B C D} \geq 0,
$$

the pattern percolates: Each and every coupling appearing on the right-hand side vanishes, $\lambda_{A B C D}(\mu)=0$, and the theory remains free at all scales [6]. Thus, we conclude that the Coleman-Gross theorem holds true for theories with Weyl fermions, and asymptotic freedom cannot be achieved without non-Abelian gauge fields.

Price of interacting fixed points.-We are now in a position to discuss the role of gauge field fluctuations for asymptotic safety and weakly coupled fixed points in general, renormalizable theories in four dimensions. At weak coupling, anomalous dimensions are small and canonical power counting remains applicable. It is then sufficient to establish weakly coupled fixed points $\left(g_{*}, Y_{*}, \lambda_{*}\right)$ for the canonically marginal couplings of the theory, which are the perturbatively controlled solutions of

$$
\left.\mu \partial_{\mu}(g, Y, \lambda)\right|_{*}=0
$$

other than the Gaussian, where at least some or all couplings are nonzero [12]. For general gauge theories, a full classification of weakly coupled fixed point solutions to (15) has
TABLE I. Fixed points of general weakly interacting quantum field theories in four dimensions. In cases (ii) and (iii), scalar selfinteractions, if present, must take fixed points $\lambda_{A B C D}^{*}$ compatible with vacuum stability [8].

\begin{tabular}{lcc}
\hline \hline Case & Condition & Fixed point \\
\hline (i) & $g_{i}=\mathbf{Y}_{J K}^{A}=\lambda_{A B C D}=0$ & Gaussian \\
(ii) & Some $g_{i} \neq 0$, all $\mathbf{Y}_{J K}^{A}=0$ & Banks-Zaks \\
(iii) & Some $g_{i} \neq 0$, some $\mathbf{Y}_{J K}^{A} \neq 0$ & Gauge-Yukawa \\
\hline \hline
\end{tabular}

been given in Ref. [8]. Perturbative fixed points are either free (the Gaussian), interacting in the gauge sector (CaswellBanks-Zaks fixed points) [35,36], or simultaneously interacting in the gauge and the Yukawa sector (gauge-Yukawa fixed points). Fixed points may be partially or fully interacting, depending on whether some or all gauge couplings take nonzero values. Scalar self-interactions must take free or interacting fixed points of their own, compatible with vacuum stability (Table I). We stress that any weakly interacting fixed point is controlled by the one-loop gauge coefficient in (3), irrespective of its sign. Its smallness for suitable matter ensures strict perturbativity $[8,12]$. BanksZaks fixed points, if they exist, are always infrared. The Gaussian and gauge-Yukawa fixed points can be infrared or ultraviolet. In particular, asymptotic safety at weak coupling arises solely via gauge-Yukawa fixed points [8]. We conclude that weakly interacting fixed points and asymptotic safety in non-Abelian gauge theories with matter have the exact same origin as asymptotic freedom.

No-go theorem for scalar-Yukawa fixed points.-In order to complete our claim, and inasmuch as asymptotic freedom cannot arise without non-Abelian gauge fields, we finally must show that weakly interacting fixed points cannot arise in the absence of gauge interactions. To that end, we return to scalar-Yukawa theories with interaction Lagrangian (1). Assuming that Yukawa and scalar couplings are small, we must have $\left.\mu \partial_{\mu} \mathbf{Y}^{A}\right|_{*}=0$ at the leading nontrivial order in perturbation theory. Consequently, the bounds (10) and (11) must be saturated. However, Eqs. (10) vanish for only vanishing Yukawa couplings:

$$
\mathbf{Y}_{J K}^{A}=0 .
$$

This is understood as follows. Being a sum of absolute values squared, the expression (8) vanishes if and only if each term in the final sum vanishes, $\mathbf{Y}_{2}{ }_{J K}^{A B}=0$. From the definition for $\mathbf{Y}_{2}$, and after contracting over scalar indices we find that the matrix $\mathbf{Y}_{2}^{F}$ also vanishes. Taking its trace $\mathbf{Y}_{2 J J}^{F}=\overline{\mathbf{Y}_{J K}^{A}} \mathbf{Y}_{J K}^{A}=0$ implies (16) and the vanishing of (8) and (9). We conclude that the only available fixed point in the Yukawa sector at one loop, without gauge fields, is the Gaussian, and it must be infrared.

Once more, scalar couplings cannot upset this conclusion: Scalar self-interactions contribute to the running of 
Yukawas starting at two loop. In principle, they could balance the one-loop Yukawa terms, provided they are parametrically larger while still remaining perturbative in their own right. For such a mechanism to be operative, some scalar quartics must take weakly interacting fixed points by themselves. Under this assumption, we can safely neglect the parametrically smaller Yukawa contributions. Let us then pick $A, B$ such that for some $C, D$ we have $\lambda_{A B C D}^{*} \neq 0$. This implies the strict inequality

$$
\sum_{C, D} \lambda_{A B C D}^{*} \lambda_{A B C D}^{*}>0,
$$

as this is a sum of squares of which at least one entry is nonzero. Combining (17) with (14), we conclude that the flows of $\lambda_{A B A B}$ and $\lambda_{A A B B}$ are strictly positive, which is in conflict with (15), and the assumption of a weakly coupled fixed point in the scalar sector cannot be maintained. This establishes that the sole perturbatively controlled fixed point is the Gaussian, which is invariably infrared. Ultimately, in any scalar-Yukawa theory, the unavailability of weakly interacting fixed points and asymptotic safety has the same origin as the unavailability of asymptotic freedom.

Discussion and outlook.-In this Letter, we have investigated free or weakly interacting fixed points of $4 d$ particle theories with gauge fields, fermions, or scalars. From the viewpoint of high-energy physics, our findings establish that asymptotic freedom and asymptotic safety are two sides of one and the same medal. Quantum fluctuations of matter fields alone, with or without photons, are incapable of generating a well-defined and predictive short-distance limit at weak coupling. Rather, the unique driver for viable ultraviolet completions-i.e., fixed points of the renormalization group with asymptotic freedom or asymptotic safety-are the fluctuations of non-Abelian gauge fields. We emphasize that Abelian gauge theories cannot generate strictly perturbative fixed points in the way non-Abelian theories can. Still, Abelian factors may change from infrared free to asymptotically free in the vicinity of partially interacting fixed points, very much like infrared free non-Abelian gauge factors in semisimple theories [8,13,14] (see also [37]). We conclude that non-Abelian gauge fields are the price for any particle theory to remain strictly perturbative and predictive at asymptotically high energies. We also note that Abelian and non-Abelian gauge theories with matter may achieve fixed points at moderate coupling, offering new directions for standard model extensions [15,38].

From the viewpoint of statistical physics, our results show that weakly coupled infrared fixed points and second-order quantum phase transitions cannot arise without gauge fields. It follows that the universality class for any such phase transition must contain non-Abelian gauge interactions as a source for conformality. This covers conventional LandauGinsburg-type phase transitions with well-defined order parameters [39], conformal phase transitions of the Kosterlitz-Thouless type or in the vicinity of fixed point mergers [40], or topological ones [41] with deconfined quantum critical points whose existence in four dimensions, once more, relates to free or interacting gauge fields [42]. Most notably, the findings of this work have shown that the set of necessary and sufficient conditions for weakly interacting fixed points, as stated in Ref. [8], is complete, opening a door for a systematic classification of critical fourdimensional theories.

In a related vein, our results have also implications for conformal field theories (CFTs) in four dimensions. Here, conditions under which scale invariance entails full conformal invariance are of particular interest [43-46]. Using techniques related to the proof of the $a$ theorem $[44,45]$, it has been demonstrated that any relativistic and unitary fourdimensional theory that remains perturbative in the ultraviolet or infrared asymptotes to a CFT [46]. Since all weakly interacting fixed points discussed in this work (Table I) belong to this category [8], we are lead to the important conclusion that elementary non-Abelian gauge fields are the price for interacting, unitary, and strictly perturbative CFTs in four dimensions. Moreover, the precise quantitative link between fixed points of the renormalization group and CFTs [47] can now be used to extract conformal data including scaling dimensions $[7,12-14]$ and structure coefficients [48]. This is complementary to the conformal bootstrap approach, which exploits representations of the conformal algebra and their short-distance behavior [49] without being sensitive to the presence or absence of gauge fields in the microscopic theory. We conclude that our results offer a direct route to identify and characterize many new CFTs in four dimensions from first principles.

It would be most useful to also clarify the availability interacting fixed points and asymptotic safety in more strongly coupled $4 d$ theories, both with and without quantized gravity [16-32]. This task, however, is much more challenging. First and foremost, the applicability of canonical power counting can no longer be taken for granted, and nonperturbative tools, such as functional renormalization, lattice simulations, supersymmetry, or other, become a necessity. Still, signatures of near-Gaussian scaling dimensions in asymptotically safe quantum gravity [21-24], on one side, and powerful weak-strong dualities such as in supersymmetric gauge theories [50], on the other, suggest that more advances can be made in the future.

Parts of this work have been supported by a studentship from the Science and Technology Research Council (STFC).

*a.bond@sussex.ac.uk

†.litim@sussex.ac.uk

[1] D. J. Gross and F. Wilczek, Phys. Rev. Lett. 30, 1343 (1973).

[2] H. D. Politzer, Phys. Rev. Lett. 30, 1346 (1973).

[3] D. J. Gross and F. Wilczek, Phys. Rev. D 8, 3633 (1973).

[4] T. P. Cheng, E. Eichten, and L.-F. Li, Phys. Rev. D 9, 2259 (1974). 
[5] N.-P. Chang, Phys. Rev. D 10, 2706 (1974).

[6] S. R. Coleman and D. J. Gross, Phys. Rev. Lett. 31, 851 (1973).

[7] D. F. Litim and F. Sannino, J. High Energy Phys. 12 (2014) 178.

[8] A. D. Bond and D. F. Litim, Eur. Phys. J. C 77, 429 (2017).

[9] D. Bailin and A. Love, Nucl. Phys. B75, 159 (1974).

[10] S. Weinberg, in General Relativity: An Einstein Centenary Survey, edited by S. W. Hawking and W. Israel (Cambridge University Press, Cambridge, England, 1979), p. 790.

[11] K. G. Wilson, Phys. Rev. B 4, 3174 (1971).

[12] A. D. Bond, D. F. Litim, G. Medina Vazquez, and T. Steudtner, Phys. Rev. D 97, 036019 (2018).

[13] A. D. Bond and D. F. Litim, Phys. Rev. D 97, 085008 (2018).

[14] A. D. Bond and D. F. Litim, Phys. Rev. Lett. 119, 211601 (2017).

[15] A. D. Bond, G. Hiller, K. Kowalska, and D. F. Litim, J. High Energy Phys. 08 (2017) 004.

[16] M. Reuter, Phys. Rev. D 57, 971 (1998).

[17] D. F. Litim, Phys. Rev. Lett. 92, 201301 (2004).

[18] M. Niedermaier, Classical Quantum Gravity 24, R171 (2007).

[19] D. F. Litim, Phil. Trans. R. Soc. A 369, 2759 (2011).

[20] D. Benedetti, P. F. Machado, and F. Saueressig, Nucl. Phys. B824, 168 (2010).

[21] K. Falls, D. Litim, K. Nikolakopoulos, and C. Rahmede, arXiv:1301.4191.

[22] K. Falls, D. F. Litim, K. Nikolakopoulos, and C. Rahmede, Phys. Rev. D 93, 104022 (2016).

[23] K. Falls, D. F. Litim, K. Nikolakopoulos, and C. Rahmede, Classical Quantum Gravity 35, 135006 (2018).

[24] K. Falls, C. R. King, D. F. Litim, K. Nikolakopoulos, and C. Rahmede, Phys. Rev. D 97, 086006 (2018).

[25] R. Percacci, An introduction to covariant quantum gravity and asymptotic Safety, in 100 Years of General Relativity (World Scientific, Singapore, 2017), Vol. 3.

[26] S. P. Robinson and F. Wilczek, Phys. Rev. Lett. 96, 231601 (2006).
[27] M. Shaposhnikov and C. Wetterich, Phys. Lett. B 683, 196 (2010).

[28] M. Niedermaier, Nucl. Phys. B833, 226 (2010).

[29] S. Folkerts, D. F. Litim, and J. M. Pawlowski, Phys. Lett. B 709, 234 (2012).

[30] P. Donà, A. Eichhorn, and R. Percacci, Phys. Rev. D 89, 084035 (2014).

[31] N. Christiansen, D. F. Litim, J. M. Pawlowski, and M. Reichert, Phys. Rev. D 97, 106012 (2018).

[32] A. Eichhorn, arXiv:1810.07615.

[33] M. E. Machacek and M. T. Vaughn, Nucl. Phys. B236, 221 (1984).

[34] M.-x. Luo, H.-w. Wang, and Y. Xiao, Phys. Rev. D 67, 065019 (2003).

[35] W. E. Caswell, Phys. Rev. Lett. 33, 244 (1974).

[36] T. Banks and A. Zaks, Nucl. Phys. B196, 189 (1982).

[37] A. Bond and D. Litim (to be published).

[38] K. Kowalska, A. Bond, G. Hiller, and D. Litim, Proc. Sci., EPS-HEP2017 (2017) 542.

[39] J. Zinn-Justin, Int. Ser. Monogr. Phys. 113, 1 (2002).

[40] V. A. Miransky and K. Yamawaki, Phys. Rev. D 55, 5051 (1997); 56, 3768(E) (1997).

[41] T. Senthil, Science 303, 1490 (2004).

[42] Z. Bi and T. Senthil, arXiv:1808.07465 [Phys. Rev. X (to be published)].

[43] J. Polchinski, Nucl. Phys. B303, 226 (1988).

[44] Z. Komargodski and A. Schwimmer, J. High Energy Phys. 12 (2011) 099.

[45] Z. Komargodski, J. High Energy Phys. 07 (2012) 069.

[46] M. A. Luty, J. Polchinski, and R. Rattazzi, J. High Energy Phys. 01 (2013) 152.

[47] J. Cardy, Scaling and Renormalization in Statistical Physics, Cambridge Lecture Notes in Physics (Cambridge University Press, Cambridge, England, 1996).

[48] A. Codello, M. Safari, G. P. Vacca, and O. Zanusso, Eur. Phys. J. C 78, 30 (2018).

[49] D. Poland, S. Rychkov, and A. Vichi, Rev. Mod. Phys. 91, 015002 (2019).

[50] N. Seiberg, Nucl. Phys. B435, 129 (1995). 